\title{
Case Series on Scrub Typhus from a tertiary care hospital of North East India
}

\author{
Salam Kenny Singh ${ }^{1}$, Ratan Ram $^{2}$, Linda Marangmei ${ }^{3}$, Gautam Chakma ${ }^{4}$ \\ ${ }^{\text {I}(D e p a r t m e n t ~ o f ~ M e d i c i n e, ~ R e g i o n a l ~ I n s t i t u t e ~ o f ~ M e d i c a l ~ S c i e n c e s, ~ I m p h a l, ~ M a n i p u r, ~ I n d i a) ~}$ \\ ${ }^{2}$ (Department of Medicine, Regional Institute of Medical Sciences, Imphal, Manipur, India) \\ ${ }_{3}^{3}$ (Department of Medicine, Regional Institute of Medical Sciences, Imphal, Manipur, India) \\ ${ }^{4}$ (Department of Medicine, Regional Institute of Medical Sciences, Imphal, Manipur, India)
}

\begin{abstract}
:
Introduction: Scrub typhus is a form of typhus caused by the intracellular parasite Orientia tsutsugamushi. The disease is endemic in many parts of India.

Materials and Methods: This study describes the clinical profile of six scrub typhus positive cases admitted in a tertiary care hospital. Complete physical examination and all routine tests were done in all cases. In all the cases, scrub typhus was diagnosed using a rapid single step immunochromatographic assay.

Results: Of the six cases three were females and three were males. Fever was the chief presenting symptom in all the cases. Other signs and symptoms were headache, myalgia, cough, hepatospleenomegaly, abdominal pain and lymphadenopathy. Only two patients had pathognomonic features of eschar and lymphadenopathy. Liver enzymes were elevated in all the patients. Hypotension, thrombocytopenia, hepatic dysfunction, hypoalbuminemia, hematuria and proteinuria were the complications observed. All the cases were successfully treated with doxycycline and there was no mortality.

Conclusion: Our report emphasizes the fact that a diagnosis of scrub typhus should be suspected when a patient presents with fever and laboratory evidence of liver dysfunction, as early detection of the disease could reduce morbidity and mortality.
\end{abstract}

Keywords: Eschar, Intracellular Parasite, Hypoalbuminemia, Liver dysfunction, Typhus

\section{Introduction}

Scrub typhus is an acute febrile illness caused by Orientia (Rickettsia) tsutsugamushi. It is an important consideration in the differential diagnosis of acute febrile illness in eastern Asia and the western Pacific region, from Korea to Australia and from Japan to India and Pakistan. O. tsutsugamushi is transmitted to humans by the bite of a larval-stage trombiculid mite or chigger. The incubation period is 5-10 days. Clinical manifestations are

fever, skin rash, eschar and varying degree of respiratory distress.[1]

Various serological tests are available for diagnosis of scrub typhus of which Weil Felix test is the most commonly used. The disease response to antibiotics is excellent.

The aim of the present study was to evaluate the clinical manifestation, laboratory findings and treatment outcomes of scrub typhus in a tertiary care setting.

\section{Methodology}

This study describes the epidemiologic and clinical profile of six scrub typhus positive patients who were admitted in Regional Institute of Medical Sciences, Imphal, Manipur, India. Complete physical examination, routine laboratory investigations like complete blood count, liver function tests, renal function test, urine analysis, peripheral smear for malarial parasite, blood culture and urine culture were done in all the patients.

In all cases diagnosis was based on detection of antibody against Orientia tsutsugamushi using a single step rapid immunochromatographic assay.

\section{Results}

A total of six patients with undiagnosed fever were included in the study and all patients tested positive for antibody against scrub typhus. Among the six patients three were males and three were females. All the patients were from rural area. The youngest patient was 15 year old while the oldest was aged 65 year. The cases were seen mainly in the months between July to October. The duration of illness before hospitalization ranged from 6-12 days with an average of 7.5 days. Average length of stay in the hospital was 5.75 days with the range of 3-9 days. 


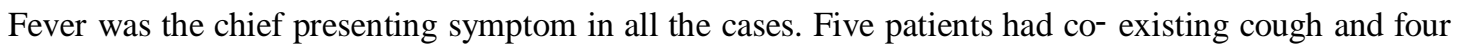
patients had headache. Myalgia was present in four cases and abdominal pain in three. Four patient had breathelessness while admission.

The pathognomonic features such as eschar and lymphadenopathy were rare in the present study, only two patients had the presence of eschar with lymphadenopathy. Groin and axilla were the observed sites of eschar.

Hepatosplenomegaly was present in four patients. Chest examination revealed crepitations in 6 cases. None of the cases in the series required intensive care unit (ICU) care. Laboratory investigations revealed anemia in four of the six cases. Total white cell counts were elevated in four cases and two had lymphocytosis. Alanine transaminase and aspartate transaminase were elevated $(>40 \mathrm{U} / \mathrm{L})$ in all the cases. Alkaline phosphatase was also elevated (>140 U/L) in all the cases. Blood cultures and urine cultures were negative in all cases.

Thrombocytopenia $(<150,000 / \mathrm{mm} 3)$ was present in three cases and hypoalbuminemia (serum albumin $<3.5 \mathrm{~g} / \mathrm{dl})$ in four cases. proteinuria.

Serum Creatinine and Urea were elevated three cases. Two patients had hematuria and five had

Hypotension was present in three cases. Laboratory evidence of hepatic dysfunction was present in all cases.

No other complications were observed in our series.

In all cases diagnosis of scrub typhus was made by a rapid single step immunochromatographic assay.

\section{Discussion}

Scrub typhus is widely endemic in Asia. In India scrub typhus has been reported in various areas especially the hilly regions of the Himalayas, Assam, Jammu \& Kashmir, West Bengal and Tamil Nadu .The causative organism is an intracellular gram-negative bacteria, Orientia tsutsugamushi. Humans are accidental hosts and the disease is transmitted through the skin by the bite of larval stage of infected trombiculid mites or chiggers.[2]

Disease occurrence is more in rainy season and occurs in persons who engage in occupational or recreational behavior that brings them into contact with mite-infested habitats.[3]

The disease presents as an acute febrile illness with non-specific signs and symptoms.[4] In our study, the commonest presentation seen was that of fever with myalgias (80\%). A necrotic eschar at the inoculating site of the mite is pathognomonic of scrub typhus[5], however, it is rarely seen in south East Asia and Indian subcontinent.[6] In our series, eschar was seen in only two patients. Though lymphadenopathy is common in scrub typhus[7], it was rare in present study. The disease usually runs a benign course but complications are not uncommon and include myocarditis, pneumonia, meningoencephalitis, gastrointestinal bleeding, acute renal failure and respiratory distress.

Serological tests still remains the main stay for the diagnosis of scrub typhus though elevated liver enzymes might give a clue. Serological test which has been widely used in India for diagnosis of scrub typhus is the Weil Felix test for it is easily available and highly specific; however it lacks sensitivity.

Another test the indirect immunofluorescence assay (IFA) is highly sensitive and considered 'gold standard' but its use is limited by the cost and availability. Microimmunofloescence, immunoperoxidase assay, latex agglutination, indirect hemagglutination, enzyme linked immunosorbent assay, dot blot immunoassay (including dipstick test) are various other serological tests available.[8] Polymerase chain reaction can detect acute infection with Orientia tsutsugamushi.[9] A rapid immunochromatographic assay which uses recombinant major outer membrane protein Antigen of Orientia tsutsugamushi to detect IgM, IgG and IgA antibodies has been shown to be reliable and suitable for use in developing countries but is expensive.[10] In the present series diagnosis in all cases was made by one step rapid immunochromatographic assay.

Doxycycline remains the antibiotic of choice for treatment of scrub typhus. Chloramphenicol, azithromycin and rifampicin are other antibiotics useful for the treatment of this infection.[11] In our series all the patients were treated with doxycycline for which they responded well and there was no mortality in our series.

\section{Conclusion}

In conclusion scrub typhus is endemic in many parts of India and all clinicians should be well aware of the disease. When a patient presents with fever and elevated liver enzymes with or without the presence of eschar, a diagnosis of scrub typhus should be considered and an empirical therapy with doxycycline should be started if there is high index of suspicion. Though eschar is pathognomonic of the disease, it may not be commonly seen, and its absence does not rule out scrub typhus. An early diagnosis \& timely antibiotic therapy may prevent further complications. 


\section{References}

[1]. Watt G, Parola P, Scrub typhus and tropical rickettsioses, Current Opinion Infectious Diseases, 16, 2003, 429-36.

[2]. P Aggarwal, Mahesh DM, Ravi Kumar V, Pratibha Himral, SS Kaushal, BS Verma, Atypical Eschar Sites in Scrub Typhus in SubHimalayas, JAPI, 57, 2009, 153.

[3]. Tilak R, Ticks and mites, in Bhalwar RV (Ed.), Textbook on public health and community medicine, 13 ( 1 st ed. Pune: Department of Community Medicine, AFMC 2009 ) 955-959.

[4]. Mahajan SK, Scrub typhus, J Assoc Physicians India, 53, 2005, 954-958.

[5]. Chogle AR, Diagnosis and treatment of scrub typhus - the 3. Indian scenario, J Assoc Physicians India, 58, 2010, 11-12.

[6]. Sharma A, Mahajan S, Gupta ML, Kanga A, Sharma V, Investigation of an outbreak of scrub typhus in the himalayan region of India, Jpn J Infect Disease,58, 2005, 208-10.

[7]. Mahajan SK, Kashyap R, Kanga A, Sharma V, Prasher BS, Relevance of Weil -Felix test in diagnosis of scrub typhus in India, J Asssoc Physicians India, 54, 2006, 619-21.

[8]. SK Mahajan, R Kashyap, A Kanga, V Sharma, BS Prasher, LS Pal, Relevance of Weil-Felix Test in Diagnosis of Scrub Typhus in India, JAPI, 54, 2006, 619-621.

[9]. Saah AJ, Orientia tsutsugamushi (scrub typhus), in Mandell GL, Bennett JE, Dolin R, (Eds.), Principles and practice of infectious disease, ( 5th ed. Philadelphia, Pa: Churchill Livingstone, 2000 ) 2056-2057.

[10]. Coleman et al, Comparative evaluation of selected diagnostic assays for the detection of IgG and IgM antibody to Orientia tsutsugamushi in Thailand, Am J Trop Med Hyg, 67, 2002, 497-503.

[11]. M Vivekanandan, Anna Mani, Yamini Sundara Priya, Ajai Pratap Singh, Samuel Jayakumar, Shashikala Purty, Outbreak of Scrub Typhus in Pondicherry. JAPI, 58, 2010, 24-28. 